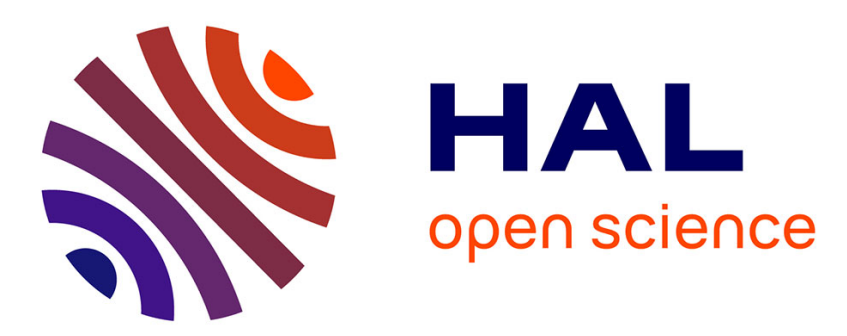

\title{
Further evidence for temporal decay in working memory: reply to Lewandowsky and Oberauer (2009).
}

Pierre Barrouillet, Sophie Portrat, Evie Vergauwe, Kevin Diependaele, Valérie Camos

\section{- To cite this version:}

Pierre Barrouillet, Sophie Portrat, Evie Vergauwe, Kevin Diependaele, Valérie Camos. Further evidence for temporal decay in working memory: reply to Lewandowsky and Oberauer (2009).. Journal of Experimental Psychology: Learning, Memory, and Cognition, 2011, 37 (5), pp.1302-17. 10.1037/a0022933 . hal-00798381

\section{HAL Id: hal-00798381 https://hal.science/hal-00798381}

Submitted on 8 Mar 2013

HAL is a multi-disciplinary open access archive for the deposit and dissemination of scientific research documents, whether they are published or not. The documents may come from teaching and research institutions in France or abroad, or from public or private research centers.
L'archive ouverte pluridisciplinaire HAL, est destinée au dépôt et à la diffusion de documents scientifiques de niveau recherche, publiés ou non, émanant des établissements d'enseignement et de recherche français ou étrangers, des laboratoires publics ou privés. 
Journal of Experimental Psychology: Learning, Memory, and Cognition, in press

\title{
Further Evidence for Temporal Decay in Working Memory
}

\author{
Pierre Barrouillet* \\ Sophie Portrat* \\ Evie Vergauwe* \\ Kevin Diependaele** \\ Valérie Camos*** \\ * Université de Genève \\ ** Ghent University \\ *** Université de Bourgogne and Institut Universitaire de France
}

Running head: Temporal decay in working memory

\section{Corresponding author:}

Pierre Barrouillet

Université de Genève

Faculté de Psychologie et de Sciences de l'Education

40, bd du pont d'Arve

1205 Genève Switzerland

Tel : 41223799251

Fax : 41223799020

Pierre.Barrouillet@unige.ch 


\begin{abstract}
The sources of forgetting in working memory are a matter of intense debate: is there a time-related decay of memory traces or is forgetting uniquely due to representation-based interference? In a previous study, we claimed to have provided evidence supporting the temporal decay hypothesis (Portrat, Barrouillet, \& Camos, 2008). However, reanalyzing our data, Lewandowsky and Oberauer (2009) demonstrated that they do not provide compelling evidence for temporal decay, and suggested a class of alternative models favoring a representation-based interference account. In this article, we develop from the most recent proposals made by these authors two of the most plausible extensions of these alternative models. We show that none of these extensions can account for recent findings related to between-domain working memory performance, and that both lead to predictions that are contradicted by new empirical evidence. Finally, we show that recent studies that have been claimed to rule out the temporal decay hypothesis do not resist close scrutiny. We conclude that the TBRS model remains the most parsimonious way to account for forgetting and restoration of memory traces in working memory.
\end{abstract}


The strong limitations on short-term memory (STM) compared to long-term memory in terms of the number of items that can be maintained and the duration of this maintenance is one of the most striking and ubiquitous phenomena of cognition. Nonetheless, the sources of forgetting over the short-term remain undecided and have recently been a matter of considerable debate. Some authors have suggested that memory traces vanish with the mere passage of time, suffering from a passive decay process (Barrouillet, Bernardin, \& Camos, 2004; Cowan \& AuBuchon, 2008; Towse \& Hitch, 1995), while others have argued that interference is the sole source of forgetting over the short term (Lewandowsky, Oberauer, \& Brown, 2009; Nairne, 1990). While recent studies on STM have favored the interference hypothesis (Berman, Jonides, \& Lewis, 2009; Lewandowsky, Duncan, \& Brown, 2004), studies on working memory (WM) using time-controlled complex span tasks seem to point to time-based decay (Barrouillet, Bernardin, Portrat, Vergauwe, \& Camos, 2007). In complex span tasks, the presentation of each item to be remembered (e.g., letters, digits, words, or locations) is followed by a processing task such as reading sentences, solving arithmetic operations or counting dots. Several studies testing our Time-Based Resource-Sharing model (TBRS) have indicated that recall performance in complex span tasks depends on a timebased parameter, named cognitive load, corresponding to the proportion of time during which the processing component of a complex span task occupies attention, thus impeding the refreshment of decaying memory traces. Of particular importance in this context were the results reported by Portrat, Barrouillet, and Camos (2008) who showed that, all other things being equal, longer processing times result in poorer recall, thus providing apparent evidence for a time decay in WM.

However, Lewandowsky and Oberauer (2009) convincingly demonstrated that this result does not constitute direct evidence for time-based forgetting in WM. They proposed an alternative account previously introduced by Lewandowsky, Oberauer, and Brown (2009) in 
which forgetting occurs through interference rather than through temporal decay. A restoration process could counteract this interference as long as attention is available. It is worth noting that this proposal does not constitute a model stricto sensu, but rather defines a class of possible models because the mechanisms of forgetting and restoration of memory traces remain unspecified. As such, it is rather difficult to test straightforward predictions issued from this proposal. This leaves us with a state of affairs in which a well-specified theory (i.e., TBRS) is opposed to an entire class of incomplete models. However, at the end of their article, Lewandowsky and Oberauer (2009) proposed to attribute forgetting to interference between representations, making reference to energy-gated encoding (Farrell \& Lewandowsky, 2002; Oberauer \& Lewandowsky, 2008), feature-sharing (Oberauer \& Kliegl, 2006), and similarity-based mechanisms (Saito \& Miyake, 2004). Following their suggestion, we considered the two accounts of representation-based interference that have been proposed by the authors themselves as possible implementations of the mechanism of forgetting, assuming that the resulting models constitute the most plausible extensions of Lewandowsky and Oberauer's proposal. In the following, we argue that none of these versions would produce what Lewandowsky and Oberauer (2009) describe as the output of this class of models. Then, we demonstrate that these versions cannot account for recent evidence in favor of temporal decay and the TBRS model, and we provide new empirical evidence in line with the TBRS model that contradicts both extensions of Lewandowsky and Oberauer's (2009) proposal. Finally, we analyze recent studies by Lewandowsky and colleagues and show that, contrary to what their authors claimed, they do not provide compelling evidence against temporal decay of the TBRS model. We conclude that, at the moment, there is still no interference-based model able to account for the findings on which the TBRS model is based.

\section{In search of evidence for temporal decay in WM tasks}


In this section, we shall briefly present the TBRS model and one of the studies conducted to demonstrate that there is a time-related decay in WM (Portrat et al., 2008), as well as the reanalysis conducted by Lewandowsky and Oberauer (2009) establishing that the results of this study do not necessarily provide evidence for temporal decay. The model that was outlined by Lewandowsky and Oberauer (2009) when accounting for our data will then be presented and compared to the TBRS model.

\section{The TBRS model}

The TBRS model stands on the idea that a central bottleneck constrains attention to alternate between processing and maintenance episodes (Barrouillet, Portrat, \& Camos, in press). One of the main constraints resulting from this sequential functioning is that, as long as attention is occupied by the processing activities, memory items suffer from a time-related decay. Thus, two factors would concur to produce forgetting in WML, interference created by the representations resulting from processing that degrades memory traces, and temporal decay of these traces when attention is occupied by processing (Barrouillet et al., 2007). However, degraded memory traces could be refreshed before their complete loss through attentional focusing. This refreshing would be achieved through a frequent and rapid switching of attention to memory traces by taking advantage of short pauses that can be freed while processing is performed. As such, in complex span tasks that combine maintenance and processing requirements, the model predicts that recall performance would be an inverse function of the proportion of time during which processing occupies attention and prevents the maintenance of memory traces. This proportion is called cognitive load, with higher cognitive load resulting in poorer recall.

We tested this prediction in several studies by presenting participants with items to be memorized, each of them being followed by a series of stimuli to process appearing successively on screen. For example, Barrouillet et al. (2004) used a reading digit span task in 
which the memoranda are series of letters of increasing length and the stimuli to be processed are digits to be read. Our model predicts that recall performance would be a function of the cognitive load of the reading digit task, which is given by the following formula

$$
\mathrm{CL}=\mathrm{aN} / \mathrm{T}
$$

in which $a$ corresponds to the duration of the attentional capture resulting from reading a digit, $N$ is the number of digits presented after each letter and $T$ the total time allowed to read them (i.e., the interval between two successive letters, Figure 1). Barrouillet et al. (2004) verified in several experiments that increasing the cognitive load of the processing task, by increasing the number of digits $N$ or by decreasing the total time $T$ allowed to read them, resulted in poorer recall performance. It appeared that WM spans are a linear function of the cognitive load involved by the reading task evaluated as the number of digits to be read per second. In a subsequent study, Barrouillet et al. (2007) tested the effects of parameter $a$. We reasoned that, all other things being equal, increasing time during which an atomic processing step captures attention would result in a more detrimental effect on concurrent maintenance. For this purpose, the digits to be read between two successive letters in the reading digit span task were presented either as words (e.g., four), Arabic digits (4), or canonical dice patterns (:). A pretest confirmed that it takes about $80 \mathrm{~ms}$ longer to identify a given number when presented in its dice-like form as compared with its word or Arabic form. As the TBRS predicted, the higher cognitive load caused by these longer reading times resulted in poorer recall in the dice pattern condition than in both the Arabic and words conditions.

In a further study that aimed at extending these findings to another process than the long-term memory retrievals involved in reading digits or identifying dice patterns, we manipulated the duration of response selections in a visuo-spatial task. Therefore, we designed a complex span task in which participants had to judge the location (up or down) of series of squares appearing successively on-screen while maintaining letters for further recall. 
The processing time was manipulated by varying the discriminability of the two locations that were either close or distant while the number of squares and the duration of the interletter interval during which they appeared were kept constant (Figures $2 \mathrm{a}$ and $\mathrm{b}$ respectively). As the TBRS model predicted, the longer processing times involved by the close condition resulted in poorer recall.

The Portrat et al. (2008) study and its re-analysis by Lewandowsky and Oberauer (2009)

Though Barrouillet et al.'s (2007) results were in line with the hypothesis that memory traces suffer from a time-related decay while concurrent processing occupies attention, Oberauer and Kliegl (2006) suggested an alternative explanation. Because the interval between two successive items to be recalled was kept constant in Barrouillet et al. (2007), any increase in the duration of processing resulted in a related decrease in the time available to refresh memory traces. This is represented in Figure $2 \mathrm{a}$ when compared with $2 \mathrm{~b}$, where lengthening the processing periods $P$ results in a shortening of the refreshing episodes $R$. Thus, the poorer recall performance observed in the close condition could have resulted from this reduction in the time available for refreshing activities and not from longer processing times resulting in more time-related decay and higher cognitive load as we hypothesized. In this case, Oberauer and Kliegl (2006) suggested that interference could have been the source of forgetting, with recall performance depending on time available for restoration. To test this alternative account, Portrat et al. (2008) used a modified task in which the response to each square was followed by a constant delay of $650 \mathrm{~ms}$ before the appearance of the next square. Thus, the close condition still involved longer processing times than the distant condition, but participants benefited in both conditions from the same time available for refreshing activities (compare Figures $2 \mathrm{~b}$ and $2 \mathrm{c}$ ). The two hypotheses of forgetting in WM led to opposite predictions. The temporal decay hypothesis still predicted a difference in recall between the 
two conditions because, even if the duration of the refreshing activities was kept constant, the close condition still involved longer processing times and thus a stronger decline of memory trace (see the evolution of memory strength in Figures $2 b$ and $2 c$ ). The interference hypothesis put forward by Oberauer and Kliegl (2006), on the other hand, did no longer predict a difference between the two conditions because recall performance is not supposed to depend on the duration of the processing episodes, but only on the time available to repair degraded memory traces, which was the same in both conditions. As a matter of fact, the close condition still resulted in poorer recall, providing strong evidence for a time-related decay of memory traces.

However, this last conclusion was probably premature. Lewandowsky and Oberauer (2009) cogently noted that the close condition not only elicited longer processing times, but also more errors ( $13 \%$ compared to $1 \%$ in the distant condition). It is known that responses following an error are slower than responses that are preceded by a correct response, a posterror slowing arising because people often self-detect their mistakes even in the absence of overt feedback. Jentzsch and Dudschig (2009) have demonstrated that this self-detection temporarily occupies the central attentional bottleneck, thus leading to a postponement of the ensuing processes and to the post-error slowing. As Lewandowsky and Oberauer (2009) noted, these post-error processes that were more frequent in the close condition probably occupied the attentional bottleneck during the restoration time following the overt response, thereby shortening the time available for refreshing memory traces (compare Figure $2 \mathrm{~d}$ and 2e). They concluded that the difference in recall performance observed between the close and the distant condition could have resulted from a reduction of the time available for restoration rather than from a longer processing time in the close condition. Re-analyzing Portrat et al.'s results, they established that when the analyses included only trials on which there were few errors on the square task (less than 16\%), there was no difference in recall performance 
between the close and distant conditions, whereas the former still involved longer response times. Lewandowsky and Oberauer (2009) concluded that there was still no evidence for time-based forgetting and suggested an alternative explanation in which forgetting would result solely from interference.

\section{Lewandowsky and Oberauer's account of forgetting in complex span tasks}

In accounting for the phenomena observed by Portrat et al (2008), Lewandowsky and Oberauer (2009, Figure 1) put forward a proposal about forgetting and restoration of memory traces in WM that was previously described in Lewandowsky, Oberauer, and Brown (2009, Figure 2). As far as we know, this proposal has never yet been developed as a model nor has it been tested. It assumes that, when maintaining items in memory for further recall, each distractor appearing on screen for processing interferes with the representation of the memory items, reducing their strength irrespective of processing duration (see Figures $2 \mathrm{~d}$ and $2 \mathrm{e}$ ). Memory traces are repaired during restoration episodes, this restoration being a function of time. This explains why memory traces can not be fully restored when post-error processing occupies part of the restoration time (Figure 2e).

This proposal differs from the TBRS model in at least two ways. First, it assumes that forgetting occurs exclusively through interference produced by distractors. The resulting reduction in memory strength is not gradual as assumed by the time-related decay hypothesis, but abrupt and quasi-immediate. Thus, the impact of processing on maintenance is independent from its duration. By contrast, the restoration process that leads to raise the strength of memory traces takes time and is more effective the longer the duration of the processing episodes. This leads to the second difference, which is that Lewandowsky and Oberauer assume that recall performance depends on the time available for restoration but not on processing time, whereas the TBRS assumes that recall performance depends on the cognitive load, which is a function of both processing and restoration times. More precisely, if 
$P T$ is the processing time and ResT the time available for restoration, cognitive load $C L$ is $P T$ $/(P T+\operatorname{Res} T)$. As we will see below, Lewandowsky and Oberauer's (2009) suggestion is a clever attempt to account for time-related phenomena in memory without assuming temporal decay. However, we will see that it is difficult to imagine a mechanism of interference that would produce the variations in memory strength described in Figure $2 \mathrm{~d}$ and $2 \mathrm{e}$ concerning the task used by Portrat et al. (2008).

\section{Is Lewandowsky and Oberauer's account plausible?}

At the first glance, Lewandowsky and Oberauer's account seems to provide a simple and elegant explanation of Portrat et al. (2008) findings. Unfortunately, things may not be so simple. As we noted above, Lewandowsky and Oberauer (2009) did not give a precise account of the mechanism whereby forgetting occurs, their proposal pointing to an entire class of models. Nonetheless, they attributed forgetting to representation-based interference, and referred to recent detailed accounts of such a mechanism of forgetting (i.e., Farrell \& Lewandowsky, 2002; Oberauer \& Kliegl, 2006; Oberauer \& Lewandowsky, 2008; Saito \& Miyake, 2004). Among these accounts, two of them have been proposed by Lewandowsky and Oberauer themselves. Namely, Oberauer and Lewandowsky (2008; see also Farrell \& Lewandowsky, 2002) assume that forgetting occurs in the short term through the interference created by energy-gated encoding, whereas Oberauer and Kliegl (2006) suggest that the mechanism responsible for forgetting is interference due to feature sharing. Thus, we will consider that introducing these mechanisms of forgetting into Lewandowsky and Oberauer's proposal provides us with its two most plausible versions. As we will see, none of these versions can account for Portrat et al. (2008) findings.

Oberauer and Lewandowsky (2008) addressed the question of forgetting in short-term memory and contrasted decay, temporal distinctiveness, and interference. They favored an interference account in which items interfere with each other by being superimposed in a 
composite memory, the more items being encoded, the more their representations being blurred, like within the SOB model (Farrell \& Lewandowsky, 2002; Lewandowsky et al. 2009). According to the SOB model, encoding is energy gated and novelty sensitive. This means that the encoding strength of each item is a function of its novelty or dissimilarity compared with the current content of short-term memory (Oberauer \& Lewandowsky, 2008). A major consequence of this energy gated encoding is that repetition of interfering information has little additional effect on memory. This property was alleged by Oberauer and Lewandowsky (2008) to account for previous results reported by Lewandowsky et al. (2004) who observed that when participants are asked to repeat an irrelevant word (super) between retrievals in an immediate serial recall task, the number of repetitions of this word has no impact on recall performance (see below for detailed discussion of these findings). Oberauer and Lewandowsky (2008, p. 546) stated that "because repetition of the same word two or three times does not involve any new representations beyond those generated for its first utterance, increasing time through repetition arguably involves very little, if any, additional interference". Thus, a single distractor would produce a large decrement of memory, but subsequent occurrences of the same distractor would have little effect, if any.

This repetition of the same distracting stimulus is exactly what occurs in Portrat et al. (2008) study. It should be remembered that the memoranda in this study were lists of letters, each letter being followed by 8 successive appearances of the same black square in two possible locations. Even if one considers that the two possible locations of this square constitute two different items ${ }^{1}$, these items are repeated over and over without any change. With 4 occurrences of each location after each letter, there were 396 occurrences of the square in each location across the 18 series of a given experimental condition. It is clear that the additional interference created by repeating these stimuli within each inter-letter interval would soon become negligible, the damages to be repaired during the successive restoration 
episodes resulting only from their first appearance. However, this does not correspond to the way Lewandowsky and Oberauer (2009) depict the evolution of memory strength through the completion of the complex span task (see Figures $2 \mathrm{~d}$ and $2 \mathrm{e}$ ), where each appearance of the distractor is assumed to involve the same and unchanging reduction in memory strength. Such a recurrent reduction can not result from interference as conceived of by Oberauer and Lewandowsky (2008) because the two items to be processed are very similar and always repeated over and over.

Would other accounts of the interference phenomenon be more convincing? Oberauer and Kliegl (2006) had previously proposed a formal model of capacity limits in WM in which interference occurs through feature sharing. When two items share features, they compete for these features that can be lost in one of the representations, resulting in degraded memory traces and forgetting. Though Oberauer and Kliegl (2006) claimed that there is a certain degree of feature overlap between any two items, Oberauer and Lange (2008) assumed that pairing verbal with visuo-spatial items would result in a minimal feature overlap and engender little interference, and sometimes none at all, as an abundant literature suggests (Cocchini, Logie, Della Sala, MacPherson, \& Baddeley, 2002; Hale, Myerson, Emery, Lawrence, \& Dufault, 2007). We agree with Oberauer and Lange (2008). Actually, it is highly difficult to envision what kind of features a black square is sharing with letters in such a way that they create interference, and how this interference could reappear at each encounter of the square after having been previously repaired, as Lewandowsky and Oberauer (2009) suggested. It could be argued that because the square task requires a decision whether the square is above or below the midline of the screen, participants must use representations of the categories "above " and "below", and it is possible that verbal labels of these categories have features that overlap with letters ${ }^{2}$. However, there is empirical evidence that the disruptive effect of the square task on verbal maintenance is not due to some verbal 
component of the spatial judgment. In a recent study, we observed that the effect of the square task on verbal maintenance remained absolutely unchanged under concurrent articulation, which is commonly used to prevent undesirable coding of spatial stimuli (Mora, Camos, and Barrouillet, 2009). This suggests that the effect of the square task on verbal maintenance does not rely on some verbal coding of the spatial judgment task. Of course, it has been shown that some form of verbal coding is still possible under articulatory suppression (Baddeley, Eldridge, \& Lewis, 1981; Besner, 1987). Thus, Mora et al. (2009) cannot provide definitive evidence that there is no verbal coding in the square task. However, it becomes increasingly difficult to understand why, despite the additional barrier of articulatory suppression, participants would persist in encoding the spatial stimuli of the square task in the precise format that has the most detrimental effect on maintenance. Thus, it remains difficult to account for the disruptive effect of the square task on the maintenance of letters through feature-sharing interference.

In summary, the interference phenomenon invoked by Lewandowsky and Oberauer (2009) in their Figure 1 and reproduced here in Figures $2 d$ and 2e to account for Portrat et al. (2008) findings does not correspond to any of the interference mechanisms these authors had previously developed (Lewandowsky, Oberauer, \& Brown, 2009; Oberauer \& Kliegl, 2006; Oberauer \& Lewandowsky, 2008). According to these mechanisms, the squares to be processed should create only a minimal amount of interference with the memoranda, and this amount would no longer increase after the first presentations of these items. Thus, the recurrent decline in memory strength that must be counteracted by restoration processes as described by Lewandowsky and Oberauer (2009) cannot result from either one of the two forgetting mechanisms these authors have recently investigated. This is not to say that there is no interference-based mechanism of forgetting that would produce the effects described in Figures $2 \mathrm{~d}$ and $2 \mathrm{e}$, but this mechanism is none of the two mechanisms previously investigated 
by Lewandowsky and Oberauer. By contrast, time-related decay can provide an explanation of what Lewandowsky and Oberauer (2009) describe in their Figure 1 because each occurrence of the square involves an analysis of its location and a response selection that temporarily occupies attention, preventing maintenance activities to take place and resulting in the decay of the memory strength. Of course, the decay predicted by the TBRS is timerelated and dependent on the duration of the attentional capture instead of immediate as in Lewandowsky and Oberauer (2009).

Similarly, the effects related to post-error processes are not at odds with our theory. By occupying attention, post-error processes lengthened the periods during which information declined in the close condition. As a consequence, they increased the cognitive load in the difficult condition, thus resulting in poorer recall. Nonetheless, the TBRS model does not account for the fact that there was no difference in recall performance between the close and distant conditions when few errors were committed, as Lewandowsky and Oberauer (2009) showed, though the former condition involved longer processing times than the latter. It is possible that when no additional attentional capture related to post error processing occurred, the difference in processing time between the close and the distant conditions was not sufficient to induce a sizeable difference in recall. As such, these findings are not in line with the TBRS predictions, but they do not constitute strong evidence for a mechanism of forgetting based on interference either. In the following sections, we will see that none of the two most plausible versions of Lewandowsky and Oberauer's (2009) proposal can account for phenomena predicted by the temporal decay hypothesis and the TBRS model, and that these versions lead to predictions contradicted by new empirical evidence.

\section{Temporal decay in between-domain dual task situations}

One of the main predictions of the TBRS model and its hypothesis of a temporal decay is that any activity that occupies attention and prevents maintenance activities would have a 
detrimental effect on concurrent storage. This hypothesis points towards between-domain effects in which attentional demanding processing of a given type of information would have a disruptive effect on the maintenance of information pertaining to another domain. Several studies have reported such between-domain effects. For example, Stevanovski and Jolicoeur (2007) observed that tone-pitch discrimination had a disruptive effect on visual memory for colored disks. Conversely, Jolicoeur and Dell'Acqua (1998) observed that maintaining three instead of one letter for further recall systematically elevated the reaction times to the tonepitch discrimination task. Consistent results were obtained by Chen and Cowan (2009) who reported a conflict between a speeded choice reaction time task on the location of a target (a red square) and the retention of verbal material such as digits presented either in visual or auditory format. However, the TBRS model goes further by predicting that, whatever the nature of the information to be processed and the representation-based it creates, WM loss would mainly depend on the duration of this intervening processing.

This was recently tested by Vergauwe, Barrouillet, and Camos (2010) who combined in complex span tasks either a visuo-spatial or a verbal processing component with a visuospatial memory task. The memoranda were series of $4 \times 4$ matrices containing one red square, each red square appearing at a different location within a series (Figure 3a). Processing components were both two-choice reaction time tasks. The visuo-spatial processing task was a spatial fit task (Roth \& Hellige, 1998; Rybash \& Hoyer, 1992) in which participants had to decide if a horizontal line fits into the gap between two dots (Figure 3b). The verbal processing component was a semantic categorization task in which participants were asked to decide if the word displayed on screen was an animal noun (Figure 3c).

The cognitive load involved by the processing components of the complex span tasks was varied in two ways, either by increasing the number of items to be processed within the interval between two successive memory items, or by decreasing the time available to process 
a given number of items. We predicted that, for both processing tasks, recall performance should be a direct function of the cognitive load involved by the processing component. As we expected, with both processing tasks, increasing cognitive load resulted in a linear decrease in recall performance. Though representation-based interference also concurs in WM loss, as we recently suggested (Barrouillet et al., 2007), it seemed to play here a negligible role. Temporal analyses revealed that cognitive load accounted for $93 \%$ of the variance in the mean spans (Figure 4). These results are perfectly in line with the hypothesis that visuospatial memory traces suffer from a time-related decay while attention is switched away and occupied by concurrent processing. How could the different versions drawn from Lewandowsky and Oberauer's (2009) proposal account for Vergauwe et al.'s (2010) findings?

One of the conundrums encountered by interference-based theories in accounting for these findings is that they assume that forgetting depends on the nature of the stimuli encoded, whereas our results indicate that processing verbal or visuo-spatial stimuli had no significantly different effects when temporal factors are controlled. Thus, to account for these findings in terms of interference-based forgetting, it should be assumed that the two processing tasks created comparable amounts of interference with the visuo-spatial memory. Considering the interference account based on feature-sharing as in Oberauer and Kliegl's (2006) formal model, it is difficult to imagine that the stimuli involved in the spatial fit task and the words involved in the semantic categorization task would share, even approximately, the same amount of features with the matrices presented for the memory task. A featuresharing model would predict more interference between two visuo-spatial tasks than between a verbal and a visuo-spatial task.

The interference account based on novelty, as put forward by Oberauer and Lewandowsky (2008) faces the same problem, but in the other way round. Whereas the verbal task involved 24 different words involving different phonological, orthographic, and semantic 
features, the 24 stimuli involved in the spatial fit task were all made of the same black line and the same two dots. Variations from one item to the other consisted only on the relative position of these constituents and on the length of the horizontal line. Thus, even if the stimuli of the spatial fit task differed from each other, it can reasonably be argued that the words presented a higher degree of dissimilarity between each other than the visuo-spatial stimuli did. As a consequence, one would expect that the verbal task, whose items involve a greater degree of novelty, would have produced a greater amount of interference. None of these phenomena was observed ${ }^{3}$.

Other findings from our laboratory strengthen this conclusion. For example, Vergauwe et al. (2009), using the same experimental approach, systematically combined visual and spatial processing with visual and spatial storage. It has been argued that visual working memory and spatial working memory involve different representations and distinct pools of resources. These assumptions are mainly based on empirical evidence provided by the selective-interference paradigm indicating that processing visual information selectively impairs visual but not (or to a lesser extent) spatial maintenance, whereas processing spatial information impairs spatial but not (or to a lesser extent) visual maintenance (Baddeley, 2007; Baddeley \& Logie, 1999; Klauer \& Zhao, 2004; Logie, 1995). However, when carefully controlling temporal factors and avoiding similarity between the stimuli to be processed and those to be maintained, we observed that variations in the cognitive load of either a visual or a spatial task had the same effect on visual and spatial maintenance. Once more, the time during which the intervening task occupied attention had a stronger effect than the nature of the representations involved in processing and storage activities, as temporal analyses revealed.

In summary, the results reported by Vergauwe et al. $(2009,2010)$ are difficult to reconcile with models that conceive forgetting in the short-term as exclusively related to representation-based interference. Even if additional studies are clearly needed, it becomes 
increasingly difficult to deny any time-related forgetting when carefully controlled experiments reveal that, in between-domain dual task situations, memory performance mainly depends on the duration of the intervening tasks. Thus though representation-based interference probably occurred in these experiments, none of the two models of forgetting recently put forward by Lewandowsky and Oberauer (Oberauer \& Kliegl, 2006; Oberauer \& Lewandowsky, 2008) seems sufficient to account for the findings reported.

\section{Testing the predictions of the interference account}

As we noted above, the proposal outlined by Lewandowsky and Oberauer (2009) leaves unspecified the mechanisms responsible for interference and restoration. Nonetheless, regardless of the nature of these mechanisms, this proposal assumes that recall performance in complex span tasks depends on two parameters. The first is the amount of interference created by the distractors, greater amount of interference resulting in greater reduction of memory strength and in poorer recalls. Importantly, Lewandowsky and Oberauer (2009) specify that the amount of interference created by the distractors would not depend on the time during which attention is occupied by processing (i.e., the processing time, $P T$ ), but on the nature of these distractors, thus reducing the class of eligible models to a restricted type of mechanisms of interference. The second is the time available for repairing the damages resulting from this interference (i.e., the restoration time, ResT), with longer ResT leading to greater repair of memory traces and better recall. At first sight, Lewandowsky and Oberauer's proposal appears as an alternative explanation of the findings supporting the TBRS model because, though they greatly differ at the theoretical level, both approaches make in many cases the same predictions.

For example, this is the case in most of our previous studies in which we manipulated the cognitive load of the processing component of working memory span tasks by varying the number of items to be processed after the presentation of each memory item or the time 
available to process them during the inter-memory-item intervals or both. Indeed, suppose that the number of distractors to be processed is increased while the total time allowed to process them is kept constant (compare panels a and $\mathrm{b}$ in Figure 5). The TBRS makes the prediction of poorer recall performance because increasing the number of distractors increases the total processing time $P T$ whereas the total time $T$ allowed to perform the task is kept constant, thus resulting in a higher cognitive load $(C L=P T / T)$. However, Lewandowsky and Oberauer's proposal makes the same prediction. A higher number of distractors to be processed over an unchanged period of time results in the reduction of ResT related to the increase in $P T(T=P T+\operatorname{Res} T)$. It could also result in a greater amount of interference as long as distractors differ from each other, for example by increasing the amount of novelty to be encoded or the probability of feature sharing. Both a shorter ResT and a higher amount of interference concur to lower recall performance. Imagine now that the number of distractors is kept constant while decreasing the total time $T$ available to process them (compare panel c to panel a in Figure 5). The TBRS predicts poorer recall resulting from an increase in $C L$, but Lewandowsky and Oberauer's proposal makes the same prediction because the amount of interference created by the distractors remains unchanged while ResT is reduced. Finally, suppose that the processing periods are prolonged by presenting more distractors at the same pace (Figure 5c and 5d). The TBRS does not predict any change in recall performance because $C L$ remains unchanged (Barrouillet et al., 2004). Nonetheless, as long as these distractors differ from each other, both interference-based versions of Lewandowsky and Oberauer's proposal could make the same prediction. The greater amount of interference that would result from processing more distractors could be counteracted by a proportional increase in the total $\operatorname{Res} T^{4}$. These similar predictions mainly result from the fact that, because $C L=P T /(P T+\operatorname{Res} T)$, the critical factor for the TBRS (i.e., $C L)$ and one of the critical factors within Lewandowsky and Oberauer's proposal (i.e., ResT) co-vary. 
However, it is possible to create experimental conditions in which the TBRS and the two most plausible versions of Lewandowsky and Oberauer's proposal make different predictions. Consider for example the aforementioned task used by Portrat et al. (2008) in which a location judgment task was combined with a letter span task. As we have seen above, both the TBRS and Lewandowsky and Oberauer's proposal predicts better recall when the pace of the task is slowed down by increasing the total time allowed to process a constant number of squares. However, suppose now that instead of processing 4 squares during an inter-letter interval $T$, participants are requested to process 8 squares presented at the same pace during twice as much time. In this case, both the total $P T$ and the total ResT would be doubled, leaving the cognitive load unchanged. As a consequence, the TBRS model would not predict any variation in recall performance. As we have seen above, Lewandowsky and Oberauer's proposal could make the same prediction as long as the distractors differ from each other. However, as we will see, things are different when the same distractor is repeated, as it is the case in the location judgment task.

Consider first that interference in Lewandowsky and Oberauer's proposal is due to feature-sharing. Processing visuo-spatial stimuli (black squares) would produce minimal interference with verbal material (letters to be maintained). As we discussed in the previous section, it is difficult to imagine what kind of features the squares and the letters could share to produce interference. Moreover, the interference being minimal, even a fast pace of the square task should be sufficient to counteract it. Then, it becomes uncertain that this account would even predict an effect of pace on recall. Consider now the version instantiating the novelty-based interference hypothesized by Oberauer and Lewandowsky (2008). As we noted above, according to this theory, repeating the same stimulus after its first encoding would only involve minimal additional interference. It can be assumed that after 4 occurrences of the same square, its further repetitions would add a negligible amount of novelty that would not 
create any further interference. Thus, extending the processing periods from 4 to 8 successive squares would leave approximately unchanged the amount of interference created, while the total time available to restore the resulting damages would strongly increase. If the squares are presented at the same pace during a doubled inter-letter interval, participants would benefit for twice as much time for restoration. Thus, the novelty-encoding version of Lewandowsky and Oberauer's proposal would predict an effect of the number of squares with the 8-square condition leading to better recall performance than the 4-square condition.

This prediction was tested in the following experiment in which participants were presented with the same location span task as in Portrat et al. (2008) in which each letter to be remembered was followed by either 4 or 8 squares presented at three different paces, thus resulting in 6 experimental conditions (Table 1). We opted for the distant condition of the square task in which the two possible locations of the square are easily distinguishable. According to the TBRS model, recall performance depends on the cognitive load the processing task involves. Thus, it predicts an effect of pace with slower paces resulting in better recall performance, but no effect of the number of squares. All the models subsumed under Lewandowsky and Oberauer's proposal assume that recall performance depends on the amount of interference and the time available for restoration. Thus, as the TBRS, they predict an effect of pace because faster paces mean shorter ResT for an unchanged amount of interference. By contrast, the novelty-encoding version also predicts a large effect of the number of squares, with better recall in the 8 -square than in the 4-square conditions, because the former involves twice as much ResT for an equivalent amount of interference, the same stimuli being repeated again and again. Furthermore, a closer look at Table 1 reveals that this experimental design permits us to contrast two conditions involving the same amount of interference, the same total ResT, but different cognitive loads. Previous studies indicating a mean location judgment of about $390 \mathrm{~ms}$, the time available to process each square in the 
three conditions of pace was designed in such a way that processing 4 squares at the slowest pace resulted in the same total restoration time as processing 8 squares at the fastest pace. The comparison between these two conditions thus constituted a further test because the noveltyencoding version does not predict any difference, whereas the TBRS model does predict better recall performance in the 4 square-slow pace condition that involves a lower cognitive load.

\section{Method}

Thirty undergraduate psychology students at the University of Geneva (23 females, mean age $=24$ years) received a partial course credit for participating. They were presented with series of 7 consonants to be remembered, each consonant being followed by either 4 or 8 black squares (side $=18 \mathrm{~mm}$ subtending 2 degrees in visual angle) successively displayed on one of two possible locations that were $68 \mathrm{~mm}$ apart (6.5 degrees in visual angle), either in the upper or the lower part of the screen. These squares appeared either at a fast, medium, or slow pace with one square every $780 \mathrm{~ms}, 980 \mathrm{~ms}$, or $1180 \mathrm{~ms}$ respectively. Four series of letters were associated with each of the six experimental conditions defined by the three paces (slow, medium, fast) and the two possible numbers of squares displayed (4 or 8), resulting in 24 series of consonants that were presented to each participant.

Each series began by a ready signal (an asterisk) centered on screen for $750 \mathrm{~ms}$, followed after a $500 \mathrm{~ms}$ delay by the first letter presented for $1500 \mathrm{~ms}$. After a post-letter delay of $500 \mathrm{~ms}$, the 4 or 8 squares of the location judgment task appeared successively on screen for $520 \mathrm{~ms}$. Each square was followed by a delay of either $260 \mathrm{~ms}, 460 \mathrm{~ms}$, or $660 \mathrm{~ms}$ for a total of $780 \mathrm{~ms}, 980 \mathrm{~ms}$, or 1180 per stimulus for the fast, medium, and slow pace conditions respectively. After the last post-stimulus delay, the following consonant appeared for $1500 \mathrm{~ms}$ and so on. At the end of the series, the word "Recall" was displayed on screen. The 24 series were presented randomly, the participant being informed about the number of 
squares and the pace of each series (e.g., "4 squares / fast"). In each condition and each series, the squares were randomly displayed in the upper and the lower locations with the same frequency. Participants were asked to read aloud each letter, to judge the location of each square as fast as possible without sacrificing accuracy by pressing either a left or a righthanded key for the lower and the upper location respectively. At the end of the series, serial recall of the letters was prompted by numbers from 1 to 7 that appeared successively on screen. Participants were asked to type the letter corresponding to the serial position indicated by the number on screen or to press the space bar when this letter was forgotten. Besides the letters recalled, response times and accuracy on the location judgment task were recorded.

A training phase preceded the experimental session in which participants were first familiarized with the location judgment task (56 stimuli in each pace condition with a $80 \%$ correct criterion requested) and then with the WM task with one series of letters in the 4square medium-pace condition and one series in the 8-square medium-pace condition.

\section{Results and discussion}

Though the rate of correct responses on the location judgment task was quite high (96\%), there was a significant effect of pace with faster pace resulting in more frequent errors (3\%, $3 \%$ and $4 \%$ of errors for the slow, medium and fast paces respectively), $F(2,58)=5.06$, $p<.01$, whereas the effect of number of squares presented and the interaction were not significant, $F s<1$. Response times were very close to the expected $390 \mathrm{~ms}$, and thus the observed total PTs and total ResTs were also very close to the expected values (see Table 1).

As far as recall performance was concerned, though total ResT was twice longer in the 8-square than in the 4-square conditions, there was no significant effect of the number of squares on the percent of letters recalled in correct position (69\% and $67 \%$ respectively), $F(1$, $29)=1.97, p>.10$. By contrast, and as the TBRS predicted, there was a significant effect of pace with faster pace resulting in lower recall performance $(72 \%, 69 \%$, and $65 \%$ for the slow, 
medium and fast paces respectively), $F(2,58)=5.84, p<.01$, with no significant interaction with the number of squares, $F<1$. The comparison between the 4 -square slow and 8 -square fast conditions, which involved the same total ResT, revealed a significant difference with better recall in the former condition involving a lower cognitive load, $F(1,29)=4.97, p<$ .05 . Thus, as we predicted, doubling $P T$ while keeping ResT constant resulted in poorer recall, whereas doubling ResT while keeping pace constant had no significant effect. In summary, none of the present results supported the novelty-encoding version of Lewandowsky and Oberauer's proposal assuming that recall performance mainly depends on the time available to restore the damages resulting from interference while the time during which attention is occupied by processing would not have any effect per se.

It is worth to note that the intervention of possible post-error processes could not explain the present results. For example, the fact that there was no significant effect of the number of squares can not be explained by more frequent post-error processing in the 8square conditions leading to a reduction in $\operatorname{Res} T$ because these conditions did not involve more errors. In the same way, the difference observed between the 8-square fast and 4-square slow conditions does not seem to rely on post-error processes. Because the former condition involved twice as much squares as the latter (the participants processed a total of 224 and 112 squares respectively) and was displayed at a faster rate, it elicited more errors (mean total number of errors of 10.67 and 3.00 respectively), $F(1,29)=15.85, p<.001$. Thus, it could be argued that the observed difference in recall performance was due to more frequent posterror processes in the square task that curtailed ResT in the 8-square fast condition. However, this is not the case. If the effect on recall performance was due to more post-error processes in the 8-square fast condition, a larger difference in errors between the 8-square fast and the 4square slow conditions would result in a stronger effect on recall. We computed for each of the 30 participants the difference in the number of errors elicited by the two conditions in the 
square task as well as the effect on recall performance. Actually, there was no significant correlation between the two measures, $r=.160, p>.10$. This result was confirmed by an analysis of variance on recall performance in which we contrasted two groups of participants as a function of their difference in the number of errors, with 15 participants whose difference was higher than the median difference (i.e., 6) and the remaining 15 participants whose difference was lower than 6 . Whereas the 8 -square fast condition elicited far more errors than the 4-square slow condition in the former group (16.07 and 3.47 respectively), this difference was strongly reduced in the latter (5.27 and 2.53 respectively). The analysis of variance revealed an effect of conditions already observed with better recall performance in the 4-slow condition, $F(1,28)=4.81, p<.05$, but there was no significant effect of group, $F(1,28)=$ $1.82, p>.10$, and more importantly no interaction, $F<1$. Indeed, the effect on recall performance did not vary between those participants who had a large difference in errors (74\% and $68 \%$ of correct recall in the 4 -square slow and 8 -square fast conditions respectively) and those who had a small difference (66\% and $61 \%$ respectively). Thus, the better recall performance in the 4-square slow condition compared with the 8-square fast condition was not due to more frequent post-error processes in the latter condition.

To illustrate the significance of the present results, it must be considered that even at the fastest pace, the 8 -square condition allowed an extra ResT of about $1.5 \mathrm{~s}$ per interletter interval compared to the 4-square condition, resulting over the 7-letter series in more than 10 s of extra time available to repair interference-related damages that are assumed to remain constant from one condition to the other. However, these $10 \mathrm{~s}$ did not have a significant effect on recall, contradicting the most plausible version of Lewandowsky and Oberauer's proposal. These results were confirmed in a mixed-effects analysis of covariance carried out with the lme4 package (Bates \& Maechler, 2010) in R (R Development Core Team, 2010). Cognitive Load, Free Time and Percent Incorrect were entered step-wise in a model with Percent 
Recall as the dependent measure, Number of Squares (4 versus 8) and Pace (slow-mediumfast) as fixed factors and Participant as random factor (nested under Number of Squares). The best fit was obtained with the model that only included Cognitive Load as a covariate (based on the Bayesian Information Criterion). There was less recall as the cognitive load increased $(\beta=-102.14,|t|=2.78, p<.01 ; p$-value obtained via Markov-Chain Monte Carlo sampled posterior density). When all three covariates were included in the model, the effect of Cognitive Load was the only significant one. None of the models showed significant main effect of Number of Squares nor did they show any interactions with this factor. Thus the results did not support the main prediction of the novelty-encoding version of Lewandowsky and Oberauer's proposal according to which longer ResT should result in better recall.

\section{Assessing the alleged direct evidence against temporal decay}

By now, we have focused on empirical findings supporting the hypothesis that memory traces suffer from a time-related decay when attention is occupied by intervening processing. However, the temporal decay hypothesis has been the object of many criticisms (Brown \& Lewandowsky, 2010; Lewandowsky, Brown, \& Oberauer, 2009). In a recent series of studies, Lewandowsky and colleagues claimed having demonstrated that there is no temporal decay in short-term and working memory (Lewandowsky, Duncan, and Brown, 2004; Lewandowsky, Geiger, Morrell, and Oberauer, 2010; Lewandowsky, Geiger, and Oberauer, 2008; Oberauer and Lewandowsky, 2008). In this section, we address these studies and discuss the alleged empirical evidence against temporal decay and the TBRS model.

In order to investigate the effect of time on immediate serial recall of letters, Lewandowsky et al. (2004) asked participants to repeat a suppressor (“super") either one, two, or three times before the recall of each letter, thus increasing the delay between study and output. The results revealed no effect of the number of suppressors uttered, suggesting that memory traces do not suffer any temporal decay. These results were replicated and extended 
by Oberauer and Lewandowsky (2008) in two ways. First, the suppressors were uttered at recall, as in Lewandowsky et al. (2004), but also at encoding as in a complex span procedure. Second, and more importantly, Oberauer and Lewandowsky (2008) acknowledged that utterance of a suppressor blocks any verbal rehearsal, but not the attentional refreshing postulated by the TBRS model. Because, according to the authors, the attentional mechanism might be engaged very little or not at all by repetitive articulation of the same word ("super"), the memory content might be attentionally refreshed. This refreshing mechanism could counteract the effect of decay, explaining why increasing the number of "super" has no effect on memory performance. In order to block this mechanism of maintenance, they added a choice reaction task to the utterance of the suppressor "super", creating two different conditions. In a first condition referred to as AS-only (for articulatory suppression only), participants were asked to utter the word "super" in response to a stimulus (an asterisk) that appeared either one or four times on screen. In a second condition referred to as AS + CRT (for articulatory suppression + choice reaction task), the stimuli were either $\&$ or $\%$. In response to these stimuli, participants had to say "super" aloud and press the left or the right key respectively. The results revealed poorer recall performance in the AS + CRT than in the AS-only condition, but no effect of the number of distractors to be processed. These results were replicated by Lewandowsky et al. (2008) who observed no difference in recall between one and four distractors in the AS + CRT condition at retrieval. Lewandowsky et al. (2008) considered that these findings make a strong case against temporal decay. Because the AS + CRT condition was considered as impeding any mechanism of maintenance of memory traces, increasing the delay between encoding and recall would have resulted in poorer recall if memory traces decayed with time.

Though these findings are considered as direct evidence against temporal decay (Brown \& Lewandowsky, 2010; Lewandowsky et al., 2009), we will see that there are not as 
compelling as their authors assume. Consider first the tasks in which the delay between encoding and recall only involves the utterance of a varying number of "super". As Oberauer and Lewandowsky (2008) acknowledged, this articulatory suppression places very little demand on the attentional bottleneck and does not block the mechanism of attentional refreshing. However, there are both behavioral (Camos, Lagner, \& Barrouillet, 2009; Hudjetz \& Oberauer, 2007) and neurological (Raye et al., 2007) evidence that attentional refreshing and verbal rehearsal are independent mechanisms that can operate jointly to maintain verbal information. Consequently, the "super" experiments can not provide any evidence against temporal decay because the utterance of the suppressor only blocks verbal rehearsal but not attentional refreshing. It is because they were aware of this limitation that Oberauer and Lewandowsky added a choice reaction task to the utterance of "super". Unfortunately, the resulting task presents an important drawback that makes it inappropriate to investigate the existence of temporal decay.

Any enquiry about the role of time in short-term and working memory would at the very least necessitate a strict control of the temporal parameters of the tasks because it is of crucial importance to control the possibility for participants to surreptitiously turn their attention towards the memoranda when processing distractors. This is why, from the beginning, we abandoned the traditional self-paced working memory span tasks to use computer-paced tasks in which participants' activities are time-constrained. However, all the tasks used by Lewandowsky and colleagues are self-paced. For example, in both the AS-only and AS + CRT conditions in Oberauer and Lewandowsky (2008), after their response to a stimulus, participants displayed on screen the next stimulus by pressing themselves the up arrow key. In Lewandowsky et al. (2008, Exp. 4), participants pressed the up arrow key to proceed the next burst of distractors after recalling a letter and, when four distractors were presented, it was the key press in response to a stimulus that displayed on screen the next one. Thus, contrary to 
Lewandowsky et al.'s (2008) claim, the CRT condition did not place continuous demands on the attentional bottleneck because participants were free to postpone the processing of the next distractor in any time all along the task. Moreover, this postponement had no effect on the time available to process the following distractor, the processing of which was also selfpaced. The mean latencies for processing distractors provided by the authors do not dispel this suspicion. In Oberauer and Lewandowsky (2008), processing a series of four distractors took 3.28 s (i.e., a mean of $820 \mathrm{~ms}$ by distractor). This pace cannot be considered as leading to a continuous occupation of the attentional bottleneck. For example, these processing times can be compared with those observed in similar tasks by Barrouillet et al. (2007) who had participants judge either the parity or the spatial location (either above or below an horizontal line centered on screen) of series of digits presented successively on screen after each letter to be remembered. In these experiments, the mean RTs were $554 \mathrm{~ms}$ for the parity task and 411 ms for the location task. Under the strongest temporal constraint created by the need to process eight stimuli during the $6400 \mathrm{~ms}$ of each interletter interval, these reaction times dropped to $483 \mathrm{~ms}$ and $361 \mathrm{~ms}$ respectively. It can be seen that these values are far from the $820 \mathrm{~ms}$ that participants took to process each distractor of the choice reaction task proposed by Oberauer and Lewandowsky (2008), whose participants most probably took time to refresh memory traces before processing each stimulus. Thus, it cannot be assumed as Oberauer and Lewandowsky (2008) did that the AS + CRT task created the continuous occupation of the attentional bottleneck needed to draw conclusions against temporal decay.

Consequently, Oberauer and Lewandowsky's (2008) results are not at odds with our model. Indeed, the TBRS assumes that recall performance depends on the pace at which distractors are processed and not on their number. At a constant pace, it predicts no effect of the number of distractors, as we observed in the present study (see also Barrouillet et al., 2004, and Plancher \& Barrouillet, 2010, for related empirical evidence). Increasing the delay 
between encoding and recall would result in poorer memory performance only when $C L$ is close to its maximum value (Barrouillet, Portrat, \& Camos, in press), a condition that is probably difficult to observe and certainly not satisfied in Oberauer and Lewandowsky (2008). As a consequence, the TBRS model is not challenged by these results.

In the same way, the TBRS model is not challenged by more recent results reported by Lewandowsky et al. (2010) who investigated the effects of the duration and type of distractors in a complex span task design. In this study, participants had to maintain consonants followed by either one or three words to be read. These latter bursts were either simple, with the same word repeated three times, or complex, three different words being presented. The words in the three-word bursts distractors appeared rapidly, one after the other at a rate of $200 \mathrm{~ms}$ per distractor. These three words remained visible until the experimenter pressed the space bar after their articulation by the participant. The results revealed that articulating a single word or a simple burst after each consonant had the same detrimental effect on recall compared with a condition without any distractor. By contrast, complex bursts led to poorer recall performance than simple bursts, articulating three different words being more damaging than repeatedly articulating three times the same word. Though these findings were in line with the predictions issued from SOB model, Lewandowsky et al. (2010) noted that the articulation times were shorter for simple than complex bursts (difference of $430 \mathrm{~ms}$ ). In order to equalize the delays between encoding and recall, they ran a final experiment in which complex bursts of three words were compared with simple bursts of not three but four identical words. This manipulation was successful in involving similar delays between the two critical conditions ( $2.42 \mathrm{~s}$ and $2.45 \mathrm{~s}$ for complex and simple bursts respectively). However, the results remained unchanged, with simple bursts yielding the same recall performance as a single distractor while the complex bursts still involved lower recall performance. Lewandowsky et al. (2010) argued that the absence of a distractor duration effect for the simple bursts constitute a 
challenge for the TBRS model because they assumed that participants articulating distractors continuously, their manipulation pushed cognitive load to the maximum level that can be achieved with speaking as a distractor activity. This maximum cognitive load would lead to more forgetting with longer delays if memory traces decayed with time. Another finding considered at odds with the TBRS model is that there was a large difference in recall performance following simple and complex bursts.

It is not difficult to see that these findings do not contradict the TBRS model in any way. As Lewandowsky et al. (2010, pp 962-963) noted, "it is important to note that the predictions of the TBRS can be tested only when two conditions are met: First, the processing of distractors must occupy the attentional bottleneck [...] Second, cognitive load must be verifiably high enough to prevent refreshing from fully compensating the effect of decay". It is thus surprising that, in the remaining of their paper, they systematically confuse articulatory suppression with cognitive load. As Oberauer and Lewandowsky (2008) themselves acknowledged, the articulatory suppression created by repeating the same word cannot block the attentional bottleneck and the mechanism of attentional refreshing. Thus, the articulation of simple bursts does not satisfy the first condition identified by Lewandowsky et al. (2010) to test the predictions of the TBRS model.

Our model also accounts for the difference observed between simple and complex bursts. Recall that words were presented at a rate of $200 \mathrm{~ms}$, these words remaining visible. Thus, in the case of simple bursts, participants could easily see at the first glance that all the words were the same. They had just to read this word and repeat it without paying further attention to the display. By contrast, complex bursts required reading three different words. Consequently, even though the level of articulatory suppression was similar between the two conditions (especially in Exp. 3 where articulation times were equalized), the attentional demand is higher for complex bursts, which require reading three instead of one word. This 
confound between novelty and attentional demand undermines Lewandowsky et al. (2010) results that could be due to the higher cognitive load involved by complex bursts. In a recent study, we removed this confound by presenting distractors in such a way that participants could not anticipate the nature of the forthcoming word. The results confirmed the TBRS predictions: when changing distractors were presented at a constant pace, their number had no effect on recall. Moreover, novelty per se had no effect. Complex bursts of 4 different distractors did not result in poorer recall than bursts of two distractors, one of them being repeated three times (Plancher \& Barrouillet, 2010). In summary, despite repeated claims (Brown \& Lewandowsky, 2010; Lewandowsky et al., 2009), there is no direct evidence against the hypothesis of a time related decay of memory traces in the short-term or against the main predictions of the TBRS model.

\section{General discussion}

In order to account for Portrat et al.'s (2008) findings without any recourse to the notion of temporal decay, Lewandowsky and Oberauer (2009; Lewandowsky et al., 2009) made a proposal that, at the first glance, is compatible with the results we observed. However, we have seen that the most plausible versions of this proposal do not produce the variations in memory strength described by Lewandowsky and Oberauer (2009). None of the two most recent accounts of interference-based forgetting put forward by these authors would produce what Lewandowsky and Oberauer (2009) described in their Figure 1 reproduced here in Figures $2 \mathrm{~d}$ and $2 \mathrm{e}$. Moreover, none of these versions seems able to account for other data from our research group demonstrating time-related effects occurring between domains (i.e., verbal, visual, or spatial). Finally, these versions lead to predictions that are not supported by the present empirical facts. Lewandowsky and Oberauer (2009) were cautious in their concluding comments and stated that the exact mechanisms by which forgetting occurs remain to be developed. The present investigation suggests that this mechanism cannot 
correspond to those they had previously investigated, whereas the hypothesis of temporal decay provides a straightforward account of our results. Moreover, we have shown that there is no compelling evidence that we should abandon the temporal decay hypothesis.

As other models of forgetting advocating the degradation of memory traces through feature-sharing as the unique source of forgetting, Oberauer and Kliegl's (2006) approach preserves its plausibility as long as it restrains itself within the boundaries of the verbal domain and avoids to give a precise description of what these features are. This kind of theory is difficult to reconcile with the facts reported here concerning between-domain interference. Indeed, it should be imagined that representations share a comparable amount of features both within and between domains, and it would need to be claimed that the time needed to repair the effects of this interference perfectly mimics the effects of a temporal decay. Of course, interference through feature-sharing probably contributes to forgetting as the welldocumented phonological similarity effect demonstrates. However, we claim that interference through feature-sharing can not be sufficient to account for all the phenomena of forgetting in the short term.

Oberauer and Lewandowsky's (2008) account of interference resulting from an energy gated and novelty sensitive encoding seemed more promising. However, one of the main assumptions of this theory is that the repetition of the same item does not lead to additional interference after its first encoding. Lewandowsky and Oberauer (2009) seem to have overlooked the fact that Portrat et al.'s (2008) study involved always the same item and that forgetting occurring through energy-gated encoding can not underpin the forgetting mechanism that they describe with each occurrence of the square producing the same amount of forgetting (see Figures 2e and 2d). Of course, it could be argued that repeating the same distractor involves some reduced but non negligible amount of interference, as Oberauer and Lewandowsky (2008) insinuate in some places. However, such an assumption would lead to 
undesirable consequences. Indeed, as stated by Oberauer and Lewandowsky (2008, p. 545), "Another consequence of this energy-gated encoding is that repetition of interfering information has little additional effect on memory; this consequence turns out to be crucial to accommodating recent data on forgetting in short-term memory". The authors are referring to Lewandowsky et al.'s (2004) results we reported and discussed above, which they consider as the strongest evidence against temporal decay. Recall that in this study the absence of any effect of the number of "super" was explained within a representation-based interference framework by the fact that repeating the same word does not involve any additional interference beyond that produced by its first articulation. In the same way, to account for the fact that one distractor disrupt recall performance but that two additional distractors have no further effect, Oberauer and Lewandowsky (2008) stated that "this effect could be explained by interference between the representation of the word "super" and the memory representations. This interference would be the same for saying "super" once or three times because, in both cases, a single additional representation is encoded into working memory" (p. 553). This kind of explanation does not leave any doubt on the fact that the energy-gated encoding hypothesis makes the strong prediction that repeating the same distractor does not create additional interference. Thus, the extension of Lewandowsky and Oberauer's proposal implementing novelty-based account of interference does not seem more convincing than that derived from Oberauer and Kliegl's feature sharing mechanism. It is worth to note that the findings that these extensions fail to account for, such as the time-related forgetting in between-domain complex span tasks or the absence of effect of the number of intervening squares to be processed in the experiment reported above, were predicted by the TBRS model.

The role that time plays in our subjective experience of memory phenomena is so pervasive and ubiquitous that trying to account for forgetting from short-term and working memory while denying any temporal decay of information is probably a fascinating and 
thrilling challenge. However, it is not because the history of science is rich in discoveries that ruled out our deepest intuitions that all of our introspective observations are necessarily wrong. In their attempt to account for the time-related phenomena occurring in WM complex span tasks, Lewandowsky and Oberauer (2009) put forward a general proposal encompassing a class of models in which forgetting occurs through interference while free time could be used to repair the damages produced by this interference. Unfortunately, when combined with the most recent elaborations of the forgetting mechanisms proposed by these authors such as feature-sharing (Oberauer \& Kliegl, 2006) and novelty-encoding (Oberauer \& Lewandowsky, 2008), this idea leads to undesirable predictions that are contradicted by facts. This does not mean that no forgetting mechanisms relying on representation-based interference would be able to instantiate Lewandowsky and Oberauer's (2009) proposal and to account for the data. Indeed, our data are not sufficient to rule all the possible extensions of the class of models subsumed by this proposal. Moreover, it must be admitted that the present as well as previous results we gathered constitute only indirect evidence of a temporal decay of memory traces. Nevertheless, the TBRS model and its hypotheses of forgetting through temporal decay and refreshing by attentional focusing provides a straightforward and parsimonious account of all the phenomena we reported here. 


\section{References}

Baddeley, A. D. (2007). Working memory, thought and action. Oxford : Oxford University Press.

Baddeley, A. D., Eldridge, M., \& Lewis, V. (1981). The role of subvocalisation in reading. Quarterly Journal of Experimental Psychology, 33A, 439-454.

Baddeley, A. D., \& Logie, R. H. (1999). Working memory : The multiple component model. In A. Miyake \& P. Shah (Eds.), Models of working memory (pp. 28-61). New York : Cambridge University Press.

Barrouillet, P., Bernardin, S., \& Camos, V. (2004). Time constraints and resource-sharing in adults' working memory spans. Journal of Experimental Psychology : General, 133, 83-100.

Barrouillet, P., Bernardin, S., Portrat, S., Vergauwe, E., \& Camos, V. (2007). Time and cognitive load in working memory. Journal of Experimental Psychology : Learning, Memory, and Cognition, 33, 570-585.

Barrouillet, P., Portrat, S., \& Camos, V. (in press). On the law relating processing to storage in working memory. Psychological Review.

Bates, D., \& Maechler, M. (2010). 1me4: Linear Mixed-Effects Models Using S4 Classes. R package version 0.999375-37. http://CRAN.R-project.org/package=lme4.

Berman, M. G., Jonides, J., \& Lewis, R. L. (2009). In Search of Decay in Verbal Short-Term Memory. Journal of Experimental Psychology : Learning Memory and Cognition, 35, 317-333.

Besner, D. (1987). Phonology, lexical access in reading, and articulatory suppression: A critical review. Quarterly Journal of Experimental Psychology, 39A, 85-94. 
Brown, G. D. A., \& Lewandowsky, S. (2010). Forgetting in memory models: Arguments against trace decay and consolidation failure. In S. Della Sala (Ed.), Forgetting (pp. 49-75). Hove, UK: Psychology Press.

Chen, Z., \& Cowan, N. (2009). How verbal memory loads consume attention. Memory \& Cognition, 37, 829-836.

Cocchini, G., Logie, R. H., Della Sala, S., MacPherson, S. E., \& Baddeley, A. D. (2002). Concurrent performance of two memory tasks : Evidence for domain-specific working memory systems. Memory \& Cognition, 30, 1086-1095.

Cowan, N., \& AuBuchon, A. M. (2008). Short-term memory loss over time without retroactive stimulus interference. Psychonomic Bulletin \& Review, 15, 230-235.

Farrell, S., \& Lewandowsk, S. (2002). An endogenous distributed model of ordering in serial recall. Psychonomic Bulletin \& Review, 9, 59-79.

Hale, S., Myerson, J., Emery, L., Lawrence, B. M., \& DuFault, C. (2007). Variation in working memory across the lifespan. In A.R.A. Conway, C. Jarrold, M.J. Kane, A. Miyake, \& J.N. Towse (eds). Variation in working memory (pp. 194-224). New York: Oxford University Press.

Jentzsch, I., \& Dudschig, C. (2009). Why do we slow down after an error? Mechanisms underlying the effects of posterror slowing. Quarterly Journal of Experimental Psychology, 62, 209-218.

Jolicoeur, P., \& Dell' Acqua, R. (1998). The demonstration of short term consolidation. Cognitive Psychology, 36, 138-202.

Klauer, K. C., \& Zhao, Z. M. (2004). Double dissociations in visual and spatial short-term memory. Journal of Experimental Psychology-General, 133, 355-381.

Lewandowsky, S., Duncan, M., \& Brown, G. D. A. (2004). Time does not cause forgetting in short-term serial recall. Psychonomic Bulletin and Review, 11, 771-790. 
Lewandowsky, S., Geiger, S. M., Morrell, D. B., \& Oberauer, K. (2010). Turning simple span into complex span: Time for decay or interference from distractors? Journal of Experimental Psychology: Learning, Memory, and Cognition, 36, 958-978.

Lewandowsky, S., Geiger, S. M., \& Oberauer, K. (2008). Interference-based forgetting in verbal short-term memory. Journal of Memory and Language, 59, 200-222.

Lewandowsky, S., \& Oberauer, K. (2009). No Evidence for Temporal Decay in Working Memory. Journal of Experimental Psychology : Learning Memory and Cognition, 35, $1545-1551$.

Lewandowsky, S., Oberauer, K., \& Brown, G. D. A. (2009). No temporal decay in verbal short-term memory. Trends in Cognitive Sciences, 13, 120-126.

Logie, R. H. (1995). Visuo-spatial working memory. Hillsdale, NJ : Lawrence Erlbaum.

Mora, G., Camos, V., \& Barrouillet, P. (2009). A developmental investigation of maintenance mechanisms in working memory through the phonological similarity effect. Paper presented at the The $16^{\text {th }}$ Meeting of the European Society for Cognitive Psychology, 2 5 September, Krakow (Poland).

Oberauer, K., \& Kliegl, R. (2006). A formal model of capacity limits in working memory. Journal of Memory and Language, 55, 601-626.

Oberauer, K., \& Lange, E. B. (2008). Interference in verbal working memory: Distinguishing similarity-based confusion, feature overwriting, and feature migration. Journal of Memory and Language, 58, 730-745.

Oberauer, K., \& Lewandowsky, S. (2008). Forgetting in immediate serial recall: Decay, temporal distinctiveness, or interference? Psychological Review, 115, 544-576. 
Plancher, G., \& Barrouillet, P. (2010, September). Does novelty encoding cause forgetting in working memory? Poster presented at the Fifth European Working Memory Symposium (EWOMS), Civita Castellana.

Portrat, S., Barrouillet, P., \& Camos, V. (2008). Time-related decay or interference-based forgetting in working memory? Journal of Experimental Psychology : Learning, Memory, and Cognition, 34, 1561-1564.

R Development Core Team (2010). R: A Language and Environment for Statistical Computing. R Foundation for Statistical Computing, Vienna, Austria. ISBN 3-90005107-0, URL http://www.R-project.org.

Roth, E. C., \& Hellige, J. B. (1998). Spatial processing and hemispheric asymmetry: Contributions of the transient/magnocellular visual system. Journal of Cognitive Neuroscience, 10, 472-484.

Rybash, J. M., \& Hoyer, W. J. (1992). Hemispheric specialization for categorical and coordinate spatial representations: A reappraisal. Memory \& Cognition, 20, 271-276.

Saito, S., \& Miyake, A. (2004). On the nature of forgetting and the processing-storage relationship in reading span performance. Journal of memory and Language, 50, 425443.

Stevanovski, B., \& Jolicoeur, P. (2007). Visual short-term memory: Central capacity limitations in short-term consolidation. Visual Cognition, 15, 532-563.

Towse, J. N., \& Hitch, G. J. (1995). Is there a relationship between task demand and storage space in tests of working memory capacity? The Quarterly Journal of Experimental Psychology, 48A, 108-124.

Vergauwe, E., Barrouillet, P., \& Camos, V. (2009). Visual and spatial working memory are not that dissociated after all : A time-based resource-sharing account. Journal of Experimental Psychology: Learning, Memory, and Cognition, 35, 1012-1028. 
Vergauwe, E., Barrouillet, P., \& Camos, V. (2010). Do Mental Processes Share a DomainGeneral Resource? Psychological Science, 21, 384-390. 
Author note

This research was supported by grants from the Swiss National Science foundation grant $N^{\circ} 100014-122626$ to Pierre Barrouillet, Fyssen foundation to Sophie Portrat, and the ANR-08-BLAN-0045-01 to Valérie Camos. 


\section{Footnotes}

1. It is even unclear that this spatial change from one location to the other results in any increase in novelty or feature sharing. Indeed, Lewandowsky and Oberauer (2009) state that difficult decisions in Portrat et al. (2008) study cause no additional interference compared with easier decisions. Because these difficult decisions resulted from reducing the distance between the two possible locations of the square, this suggests that the amount of interference created by the square does not depend on its location. Thus, it can be considered that distractors in Portrat et al. (2008) task consisted in the repetition of one and the same stimulus.

2. We thank Steve Lewandowsky and Klaus Oberauer for making us aware of this possibility.

3. Lewandowsky, Geiger, and Oberauer (2008) are cautious in claiming that it is unclear that the effects of within-burst similarity advocated in the verbal domain also arise with spatial interference tasks, and suggest that it remains to be ascertained by future research whether that interference is modulated by the similarity among spatial distractors. If it turned out that interference created by spatial distractor was not modulated by similarity, Oberauer and Lewandowsky's (2008) account of forgetting, which is based on energy gating and novelty sensitivity, would be restricted to verbal material. As a consequence, Oberauer and Lewandowsky's model would restrict itself to some phonological loop and the welldocumented effects related to phonological similarity. Such a model would not be able to account for the increasing number of studies demonstrating time-related between-domain effects in complex span tasks as predicted by the TBRS, which has successfully been extended to the visuo-spatial domain (Vergauwe, Barrouillet, \& Camos, 2009, 2010). Even if the forgetting mechanism advocated by Lewandowsky and Oberauer is still unspecified, current data suggest that generality across domains is a desirable characteristic. 
4. However, this latter point remains unclear. On the one hand, we could imagine that, if some equilibrium is reached between the amount of interference created by each distractor and the efficiency of the restoration allowed by the following free time, lengthening the processing periods by presenting more distractors at the same pace would not affect maintenance and recall performance. On the other hand, it could also be argued that if the inter-distractor intervals are not sufficient to completely restore the memory traces, some part of the interference created by a distractor remains when the following appears. Thus, adding distractors while keeping the pace constant (i.e., without modifying the free time between successive distractors) would recursively increase the amount of interference, thus leading to more forgetting and poorer recall with longer processing periods. Although this second option is theoretically sound and fits with Lewandowsky and Oberauer (2009, Figure 1d, reproduced here as Figure 2e), the first option should be preferred because we have already demonstrated that increasing the number of distractors while keeping processing pace constant has no effect on working memory spans, as the TBRS predicts (Barrouillet et al., 2004, Exp. 3). Thus, the class of eligible models should be further constrained to mechanisms of forgetting and restoration permitting to reach such an equilibrium. 
Table 1: Parameters of the 6 experimental conditions of the location span task and percent of letters recalled in correct position. Total PT expected and Total ResT expected are estimates based on the assumption that the processing time for one square is $390 \mathrm{~ms}$. PT: Processing Time; ResT: Restoration time, CL: Cognitive load.

\begin{tabular}{|c|c|c|c|c|c|c|c|c|}
\hline $4-$ medium pace & $990 \mathrm{~ms}$ & $3960 \mathrm{~ms}$ & $1560 \mathrm{~ms}$ & $1552 \mathrm{~ms}$ & $2400 \mathrm{~ms}$ & $2408 \mathrm{~ms}$ & .39 & 68 \\
\hline 4 - slow pace & $1190 \mathrm{~ms}$ & $4760 \mathrm{~ms}$ & $1560 \mathrm{~ms}$ & $1600 \mathrm{~ms}$ & $3200 \mathrm{~ms}$ & $3160 \mathrm{~ms}$ & .34 & 70 \\
\hline 8 - fast pace & $790 \mathrm{~ms}$ & $6320 \mathrm{~ms}$ & $3120 \mathrm{~ms}$ & $3168 \mathrm{~ms}$ & $3200 \mathrm{~ms}$ & $3152 \mathrm{~ms}$ & .50 & 65 \\
\hline 8 - medium pace & $990 \mathrm{~ms}$ & $7920 \mathrm{~ms}$ & $3120 \mathrm{~ms}$ & $3112 \mathrm{~ms}$ & $4800 \mathrm{~ms}$ & $4808 \mathrm{~ms}$ & .39 & 70 \\
\hline
\end{tabular}




\section{Figure captions}

Figure 1: Succession of the events occurring on screen in the reading digit span task, with letters to be memorized and digits to be read. T refers to the inter-letter interval.

Figure 2: Timeline of the events occurring during a processing episode between two tobe-remembered letters in a complex span task as used by Portrat et al. (2008). Panels A and B represent the difficult and easy processing conditions, respectively, with close and distant locations of the square as used in the Barrouillet et al. (2007) study. Grey and white bars represent processing $(\mathrm{P})$ and restoration $(\mathrm{R})$ times, respectively. Panel $\mathrm{C}$ represents the condition designed by Portrat et al. (2008) in which the close locations involve longer processing times whereas restoration times are equated (compare panels B and C). Below the events is shown the assumed evolution of the activation level of the first letter according to the TBRS model. Memory traces decay with time when processing occupies attention and are reactivated during restoration time. When processing takes longer (panels C vs. B), memory traces decay more whereas the same amount of restoration is allowed by equivalent restorations times. Panels D and E illustrate the alternative explanation put forward by Lewandowsky and Oberauer (2009) in which there is no temporal decay. Each square interferes with the memoranda, reducing its strength independent of processing duration, whereas memory traces can be repaired during restoration time. In panel E, difficult processing involves longer processing times but also more errors. The black bars represent post error $(\mathrm{PE})$ processes that take place after processing and that reduce restoration times. The remaining, shorter, restoration times are not sufficient to repair memory traces in the same extent as in panel $\mathrm{D}$, hence a lower activation level and poorer recall performance.

Figure 3: Stimuli used in the Vergauwe, Barrouillet, and Camos (2010) study, with examples of the memory items (Panel A), the stimuli of the visuo-spatial processing task (Panel B) and the words used for the verbal processing task (Panel C). 
Figure 4: Mean working memory spans as a function of the cognitive load involved by the verbal and the visuo-spatial processing tasks in the Vergauwe, Barrouillet, and Camos (2010) study, with the cognitive load - span regression line.

Figure 5: Timeline of the events occurring during a complex span task in which memory items (M) are followed by successive distractors (D) to be processed when the number of distractors and the time allowed to process them are manipulated. Grey and white bars represent processing $(\mathrm{P})$ and restoration $(\mathrm{R})$ times, respectively. Assuming that Panel A represents some baseline, Panel B illustrates a situation in which the number of distractors to be processed within each inter-memory-item interval is increased while the total time allowed to process them is kept constant. In Panel C, the number of distractors to be processed is kept constant compared to A, but the total time allowed to process them has been reduced. Panel D represents a task in which the number of distractors is increased compared to Panel $\mathrm{C}$, but the pace at which these distractors are displayed is kept constant, resulting in more distractors to be processed, but also in a longer total restoration time $(\Sigma \mathrm{R})$. Note that cognitive load remains constant between $\mathrm{C}$ and $\mathrm{D}$. 
47

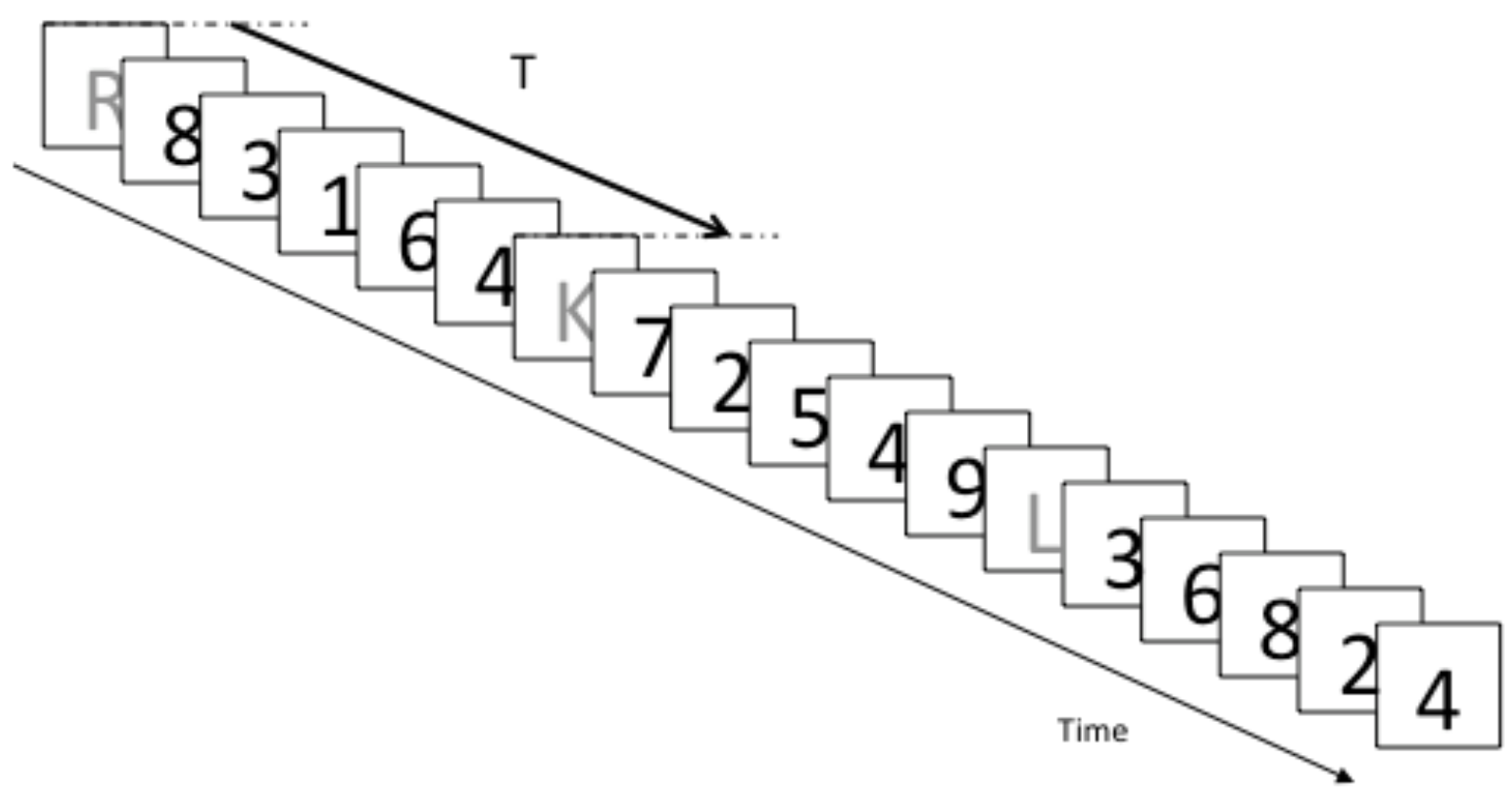

Figure 1 
A

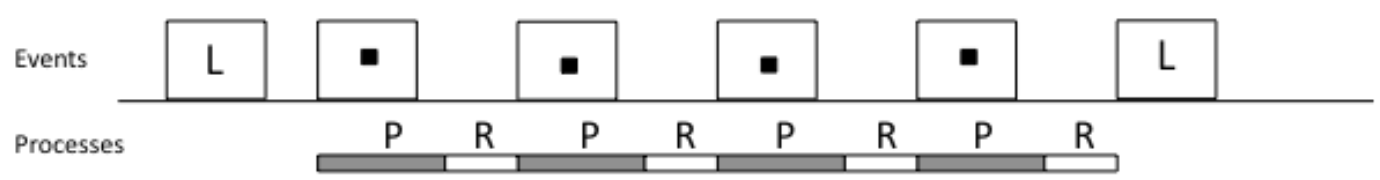

B

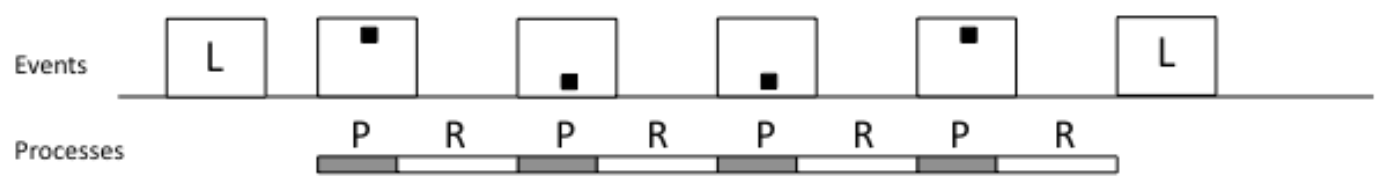

Memory

strength

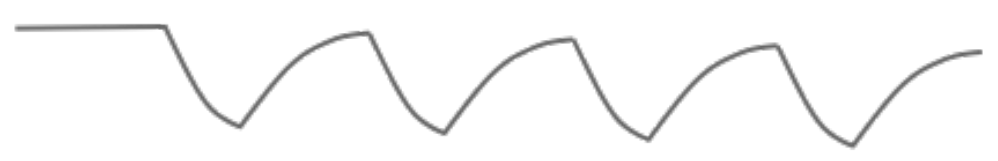

C

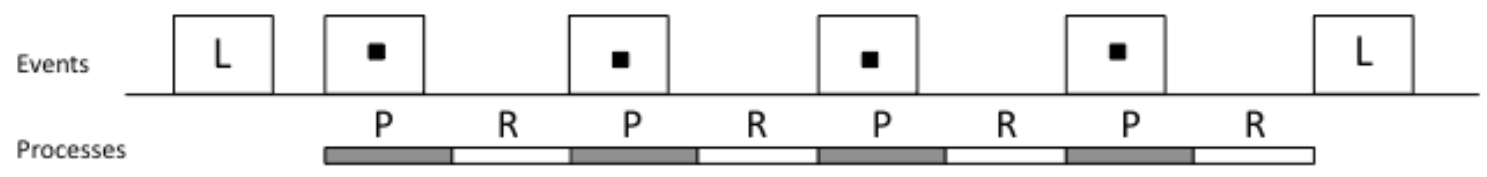

Memory

strength

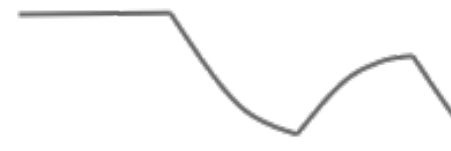

D

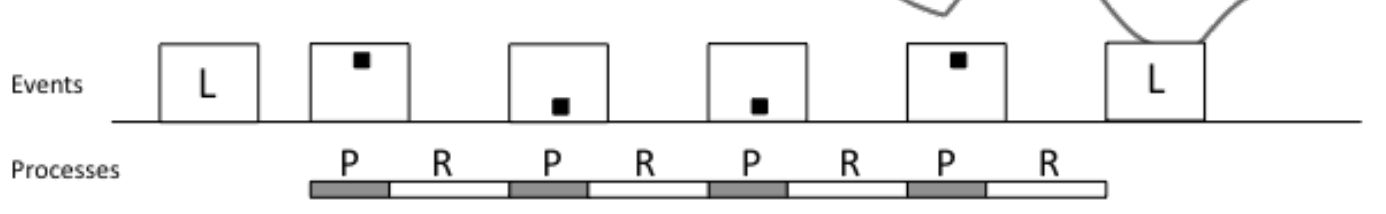

Memory

strength

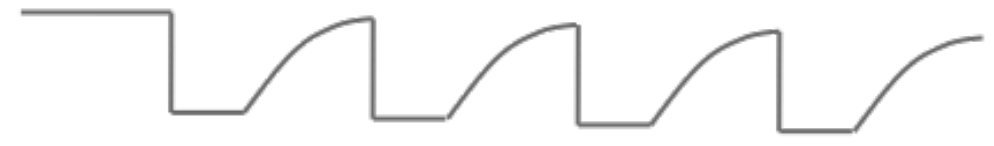

E

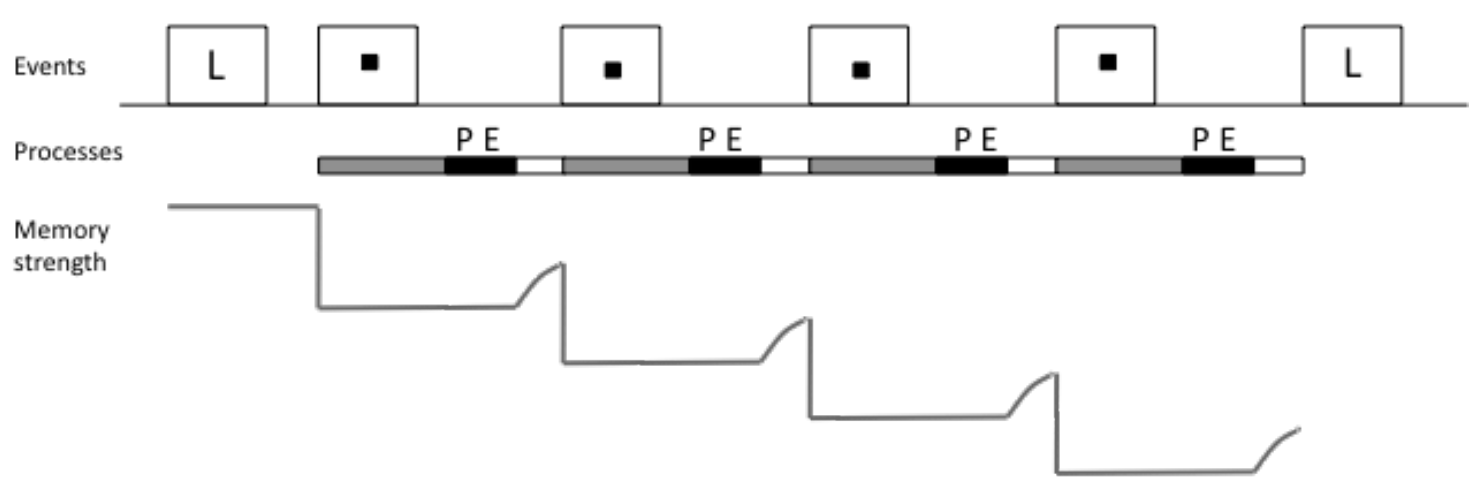

Figure 2 
A
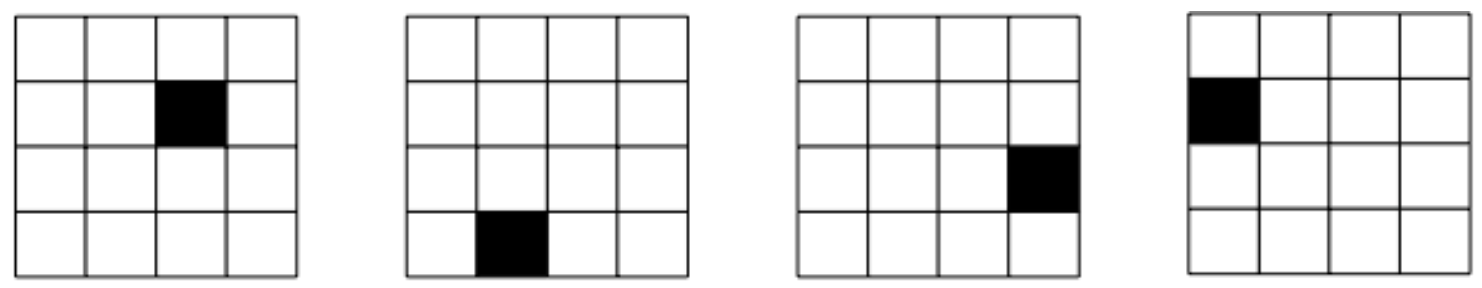

B
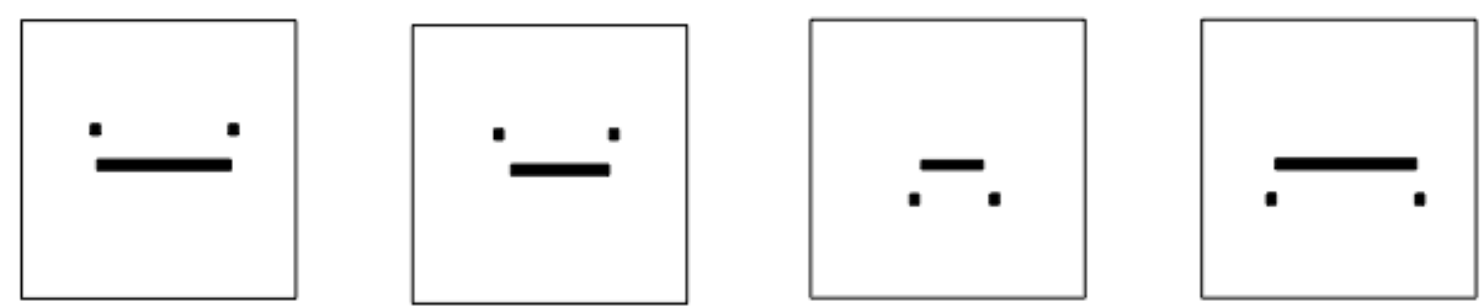

C
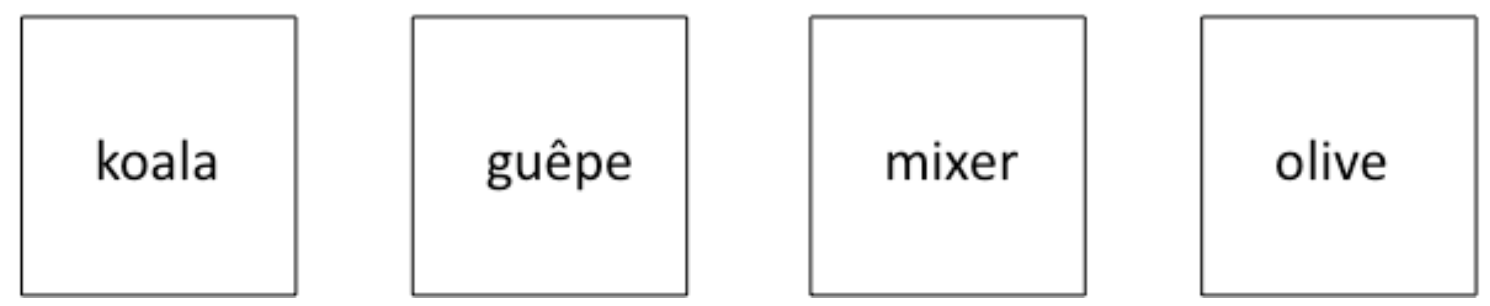

Figure 3 


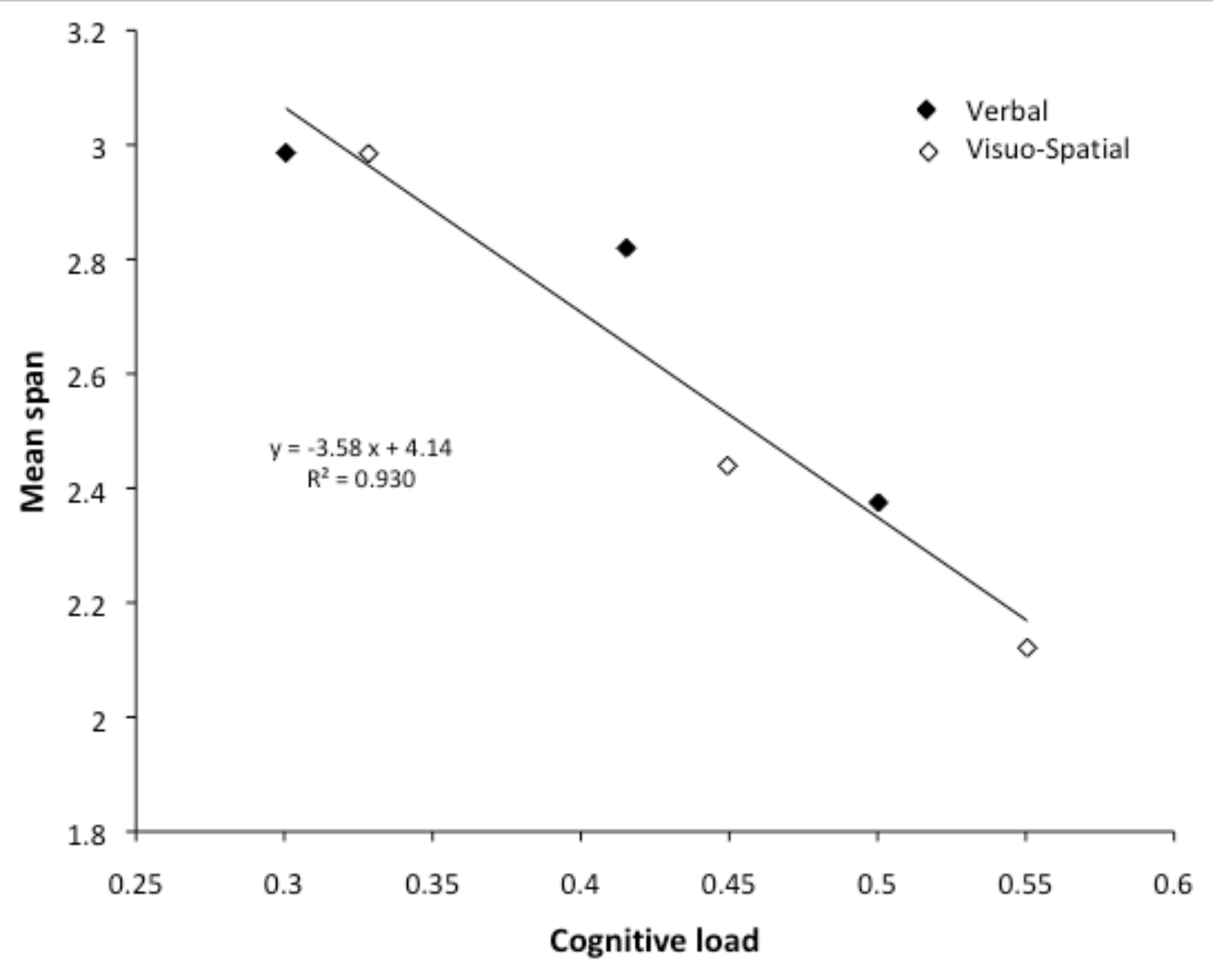

Figure 4 
A

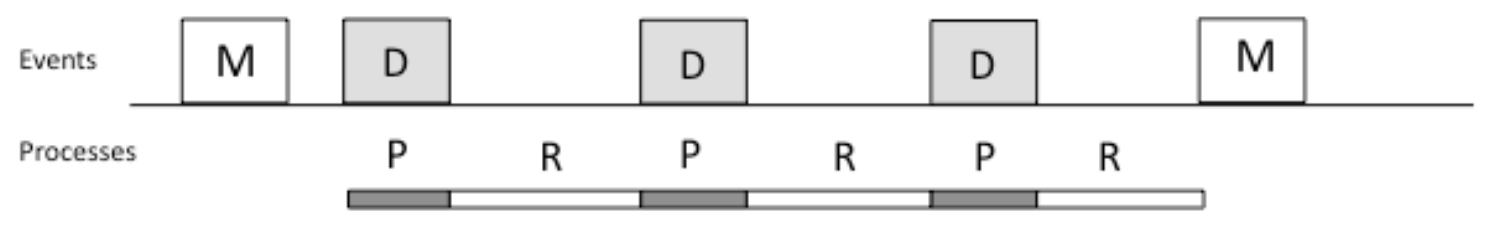

B

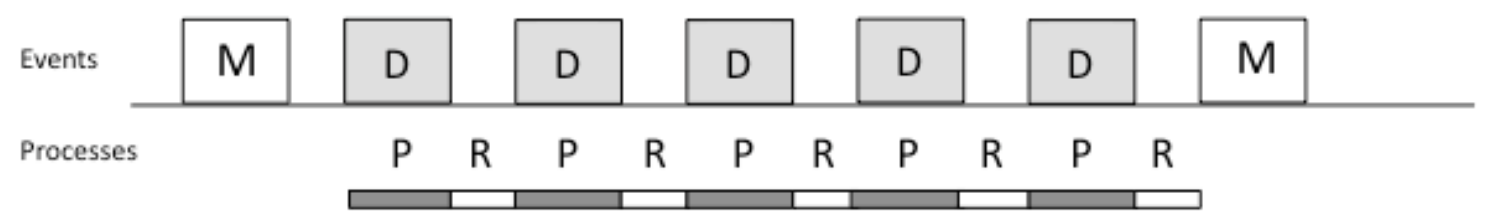

C

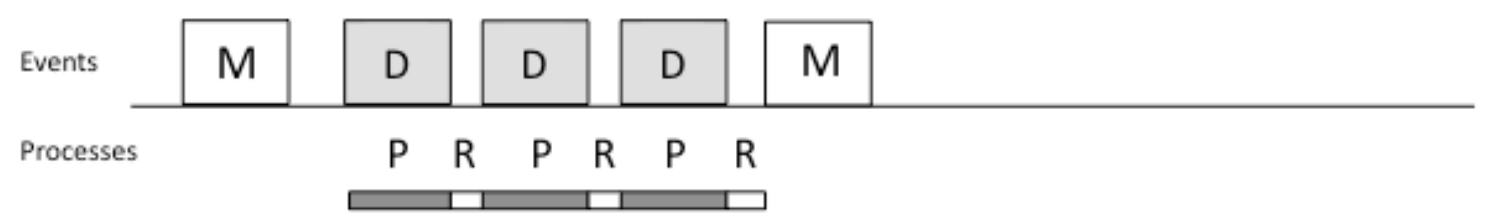

D

\begin{tabular}{|c|c|c|c|c|c|c|c|c|c|c|c|c|}
\hline Events & $\mathrm{M}$ & $D$ & D & & D & & D & & $\mathrm{D}$ & & D & $\mathrm{M}$ \\
\hline Process & & P & $P$ & $\mathrm{R}$ & P & $\mathrm{R}$ & P & $\mathrm{R}$ & P & $\mathrm{R}$ & P & $\mathrm{R}$ \\
\hline
\end{tabular}

Figure 5 